\title{
Favourable effects of the Dietary Approaches to Stop Hypertension diet on glucose tolerance and lipid profiles in gestational diabetes: a randomised clinical trial
}

\author{
Zatollah Asemi ${ }^{1}$, Zohreh Tabassi ${ }^{2}$, Mansooreh Samimi ${ }^{2}$, Taherh Fahiminejad ${ }^{2}$ \\ and Ahmad Esmaillzadeh ${ }^{3,4 *}$ \\ ${ }^{1}$ Research Center for Biochemistry and Nutrition in Metabolic Diseases, Kashan University of Medical Sciences, Kashan, Iran \\ ${ }^{2}$ Department of Gynecology and Obstetrics, School of Medicine, Kashan University of Medical Sciences, Kashan, Iran \\ ${ }^{3}$ Food Security Research Center, Isfahan University of Medical Sciences, Isfahan, Iran \\ ${ }^{4}$ Department of Community Nutrition, School of Nutrition and Food Science, Isfahan University of Medical Sciences, Isfahan \\ PO Box 81745-151, Iran
}

(Submitted 16 April 2012 - Final revision received 15 August 2012 - Accepted 16 August 2012 - First published online 13 November 2012)

\begin{abstract}
Although gestational diabetes mellitus (GDM) is associated with an increased risk of maternal and neonatal morbidity, there is no consensus as to the optimal approach of nutritional management in these patients. The present study was designed to assess the effect of the Dietary Approaches to Stop Hypertension (DASH) eating plan on glucose tolerance and lipid profiles of pregnant women with GDM. The present randomised controlled clinical trial was performed among thirty-four women diagnosed with GDM at 24-28 weeks of gestation. Subjects were randomly assigned to consume either the control diet $(n 17)$ or the DASH eating pattern ( $n$ 17) for 4 weeks. The control diet was designed to contain $45-55 \%$ carbohydrates, $15-20 \%$ protein and $25-30 \%$ total fat. The macronutrient composition of the DASH diet was similar to the control diet; however, the DASH diet was rich in fruits, vegetables, whole grains and low-fat dairy products, and contained lower amounts of saturated fats, cholesterol and refined grains with a total of $2400 \mathrm{mg}$ Na/d. Fasting blood samples were taken at baseline and after 4 weeks of intervention to measure fasting plasma glucose, glycated Hb (HbA1c) and lipid profiles. Participants underwent a $3 \mathrm{~h}$ oral glucose tolerance tests and blood samples were collected at 60, 120 and 180 min to measure plasma glucose levels. Adherence to the DASH eating pattern, compared with the control diet, resulted in improved glucose tolerance such that plasma glucose levels reduced at $60\left(-1.86 v .-0.45 \mathrm{mmol} / 1, P_{\text {group }}=0.02\right), 120\left(-2.3 v \cdot 0 \cdot 2 \mathrm{mmol} / 1, P_{\text {group }}=0.001\right)$ and $180 \mathrm{~min}(-1 \cdot 7 v$. $\left.0.22 \mathrm{mmol} / 1, P_{\text {group }}=0.002\right)$ after the glucose load. Decreased HbA1c levels $\left(-0.2 v \cdot 0.05 \%, P_{\text {group }}=0.001\right)$ was also seen in the DASH group compared with the control group. Mean changes for serum total $\left(-0.42 v \cdot 0.31 \mathrm{mmol} / \mathrm{l}, P_{\text {group }}=0 \cdot 01\right)$ and LDL-cholesterol $(-0 \cdot 47$ $\left.v .0 .22 \mathrm{mmol} / 1, P_{\text {group }}=0.005\right)$, TAG $\left(-0.17 v .0 .34 \mathrm{mmol} / 1, P_{\text {group }}=0.01\right)$ and total:HDL-cholesterol ratio ( $-0.6(\mathrm{sD} 0.9) v .0 .3(\mathrm{sD} 0.8)$, $\left.P_{\text {group }}=0.008\right)$ were significantly different between the two diets. Additionally, consumption of the DASH diet favourably influenced systolic blood pressure $\left(-2.6 v \cdot 1.7 \mathrm{mmHg}, P_{\text {group }}=0 \cdot 001\right)$. Mean changes of fasting plasma glucose $\left(-0 \cdot 29 v \cdot 0 \cdot 15 \mathrm{mmol} / 1, P_{\text {group }}=0 \cdot 09\right)$ were nonsignificant comparing the DASH diet with the control diet. In conclusion, consumption of the DASH eating pattern for 4 weeks among pregnant women with GDM resulted in beneficial effects on glucose tolerance and lipid profiles compared with the control diet.
\end{abstract}

Key words: Dietary Approaches to Stop Hypertension: Glucose tolerance: Lipid profiles: Gestational diabetes mellitus: Pregnant women

Gestational diabetes mellitus (GDM) is defined as a disorder in glucose tolerance with onset or first recognition during pregnancy ${ }^{(1)}$. It affects $5-10 \%$ of Asian pregnant women, with an increasing trend in some developing countries ${ }^{(2)}$. Several contributing factors to GDM have been found, including severe obesity during pregnancy, strong family history of type 2 diabetes (T2D) and previous history of GDM, impaired glucose metabolism or glucosuria ${ }^{(3)}$. Pregnant women with GDM are at increased risk of fetal macrosomia, prematurity, birth trauma, fetal death and respiratory distress syndrome, as well as maternal morbidity ${ }^{(4-8)}$. Also, women with a history of GDM are at increased risk of developing T2D later in life ${ }^{(9)}$.

Although several strategies have been suggested, dietary intervention is the first-line therapy for maintaining maternal

Abbreviations: DASH, Dietary Approaches to Stop Hypertension; GDM, gestational diabetes mellitus; GI, glycaemic index; HbA1c, glycated Hb; T2D, type 2 diabetes. 
postprandial glucose levels and GDM management. Earlier studies have shown the efficacy of carbohydrate restriction on metabolic control ${ }^{(10)}$ and pregnancy outcomes ${ }^{(11)}$ in GDM. In addition, low-glycaemic-index (GI) diet has also been argued as a possible strategy in GDM management ${ }^{(12)}$. Consumption of a low-GI diet among women with GDM significantly reduced the number needing to use insulin; however, no significant effects on pregnancy outcomes were found ${ }^{(13)}$.

The Dietary Approaches to Stop Hypertension (DASH) eating plan is a low-GI low-energy-dense diet that was initially suggested for lowering blood pressure ${ }^{(14)}$; however, its effectiveness has also been reported in $\mathrm{T}_{2} \mathrm{D}^{(15)}$ and the metabolic syndrome ${ }^{(16)}$. We are aware of no study examining the effect of the DASH diet on metabolic profile in GDM. High contents of dietary fibre, phyto-oestrogens and isoflavones, as well as its low $\mathrm{GI}^{(15,16)}$, might help GDM patients to control their metabolic profile. The present study was, therefore, performed to investigate the effects of the DASH eating plan on glucose tolerance and lipid profiles of pregnant women with GDM.

\section{Subjects and methods}

\section{Participants}

The present two-arm parallel randomised controlled clinical trial was carried out in Kashan, Iran, from April 2011 to December 2011. With the exception of the study dietitian (Z. A.), who provided the dietary education, all study personnel and participants were blinded to the dietary assignment. Pregnant women aged 18-40 years, diagnosed with GDM by a $100 \mathrm{~g}$ oral glucose tolerance test at 24-28 weeks of gestation, were recruited to the present study. Gestational age was assessed from the date of last menstrual period and concurrent clinical assessment ${ }^{(17)}$. Considering a type I error of $5 \%(\alpha=0.05)$, study power of $80 \%$ and serum HDLcholesterol levels as a key variable, we reached a sample size of sixteen persons for each group. Pregnant women without a previous diagnosis of glucose intolerance were screened for GDM by two procedures. First, a $50 \mathrm{~g}$ glucose challenge test was used as preliminary screening. Individuals with $1 \mathrm{~h}$ plasma glucose concentrations of $>7.77 \mathrm{mmol} / 1$ (1400 mg/l) were then asked to participate in a $100 \mathrm{~g}$ oral glucose tolerance test. Diagnosis of GDM was based on the criteria set by the American Diabetes Association ${ }^{(18)}$ and those in whom the plasma glucose levels met two of the following criteria were considered as having GDM: fasting $>5.27 \mathrm{mmol} / 1$ (950 mg/l), $1 \mathrm{~h} \geq 9.99 \mathrm{mmol} / \mathrm{l}(1800 \mathrm{mg} / \mathrm{l}), 2 \mathrm{~h} \geq 8.60 \mathrm{mmol} / \mathrm{l}$ $(1550 \mathrm{mg} / \mathrm{l})$ and $3 \mathrm{~h} \geq 7.77 \mathrm{mmol} / \mathrm{l}(1400 \mathrm{mg} / \mathrm{l})$. A total of forty-five women who attended maternity clinics affiliated to the Kashan University of Medical Sciences, Kashan, Iran, were screened for GDM, of whom forty met the inclusion criteria. We excluded those with untreated hypothyroidism, smoking and kidney or liver diseases, as well as those taking oestrogen therapy. A total of forty pregnant women were recruited in the study, and after stratification for BMI ( $<30$ and $\geq 30 \mathrm{~kg} / \mathrm{m}^{2}$ ) and weeks of gestation $(<26$ or $\geq 26$ weeks), they were randomly assigned to the control ( $n$ 20) or the DASH diet ( $n$ 20) for 4 weeks. Random assignment was done by the use of computer-generated random numbers. Among individuals in the control diet, three women (preeclampsia ( $n$ 2) or need to commence insulin therapy $(n 1)$ ) were excluded. A total of three women were excluded from the DASH diet (pre-eclampsia ( $n$ 2) and bed rest $\left(\begin{array}{ll}n & 1\end{array}\right)$ ). Finally, thirty-four participants (control diet $(n$ 17) and the DASH diet $(n$ 17)) completed the trial (Fig. 1). The study was conducted according to the guidelines laid down in the Declaration of Helsinki. The ethical committee of the Kashan University of Medical Sciences approved the study (no. 1384-90-5-18) and an informed written consent was obtained from all participants.

\section{Study design}

Participants were randomly assigned to the control or the DASH diet for 4 weeks. They were asked not to alter their routine physical activity and not to receive any lipid-lowering medications during the 4-week intervention. All pregnant women consumed a supplement of $\mathrm{Ca}$ and ferfolic once a day. Compliance with the consumption of diets was monitored once a week through phone interviews. The compliance was also double-checked by the use of $3 \mathrm{~d}$ dietary records completed throughout the study. To obtain nutrient intakes of participants based on these $3 \mathrm{~d}$ food diaries, we used Nutritionist IV software (First, Databank) modified for Iranian foods.

\section{Diets}

The control diet was designed to contain 45-55\% carbohydrates, $15-20 \%$ protein and $25-30 \%$ total fat. This diet was planned as a $7 \mathrm{~d}$ menu cycle based on the usual practice in GDM. The macronutrient composition of the DASH diet was similar to the control diet; however, the DASH diet was rich in fruits, vegetables, whole grains, low-fat dairy products, and was low in saturated fats, cholesterol, refined grains and sweets. The amount of Na intake was $2400 \mathrm{mg} / \mathrm{d}$ (Table 1$)^{(16)}$

\section{Assessment of anthropometric measures}

Maternal weight was assessed at baseline and after 4 weeks of intervention in maternity clinics by trained midwives. Body weight was measured in an overnight fasting state, without shoes and in a minimal clothing state, by the use of a digital scale (Seca) to the nearest $0 \cdot 1 \mathrm{~kg}$. Height was measured using a non-stretched tape measure (Seca) to the nearest $0 \cdot 1 \mathrm{~cm}$. BMI was calculated as weight in $\mathrm{kg}$ divided by height in $\mathrm{m}^{2}$. Pre-pregnancy weight and BMI were gathered from their earlier records.

\section{Biochemical assessment}

Fasting blood samples $(10 \mathrm{ml})$ were taken at the baseline and after the 4-week intervention at the Kashan reference laboratory early in the morning after overnight fasting. Plasma glucose levels were quantified by the use of 


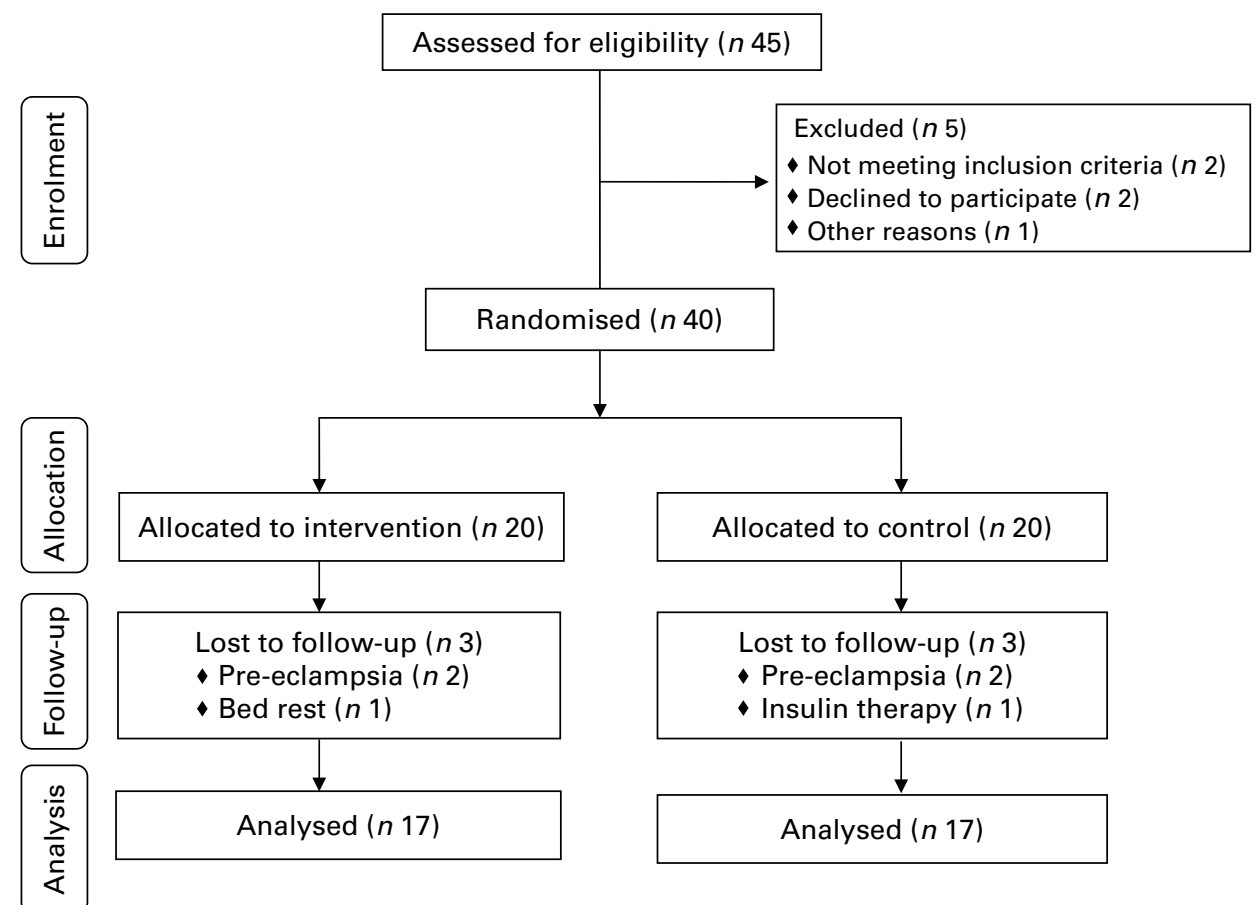

Fig. 1. Summary of patient flow.

the glucose oxidase/peroxidase (GOD-POD) method with commercially available kits (Parsazmun Company). Plasma glycated $\mathrm{Hb}$ (HbA1c) levels were measured using Glycomat kits (Biocode Hycel) by the immunoassay method. Serum total cholesterol and TAG concentrations were assayed using commercial kits (Parsazmoon Company) by enzymatic colorimetric tests containing cholesterol oxidase/p-aminophenazone and glycerol phosphate oxidase, respectively. Serum HDL-cholesterol was measured after precipitation of the apoB-containing lipoproteins with phosphotungstic acid. Serum LDL-cholesterol levels were also measured using available kits. Plasma Hb levels were measured by colorimetery (Sysmex, Model XS 8001) using the enzyme kit (Solpholizer SLS). Participants underwent $3 \mathrm{~h}$ oral glucose tolerance tests, and blood samples were collected at 60, 120 and $180 \mathrm{~min}$ to measure plasma glucose levels.

Table 1. Constituents of the Dietary Approaches to Stop Hypertension (DASH) and control diets used in the study*

\begin{tabular}{lcc}
\hline Food group & Control diet & DASH diet \\
\hline Grains $†$ & 12 & 9 \\
Simple sugars & 3 & 1 \\
Vegetables & 3 & 5 \\
Fruits & 3 & 6 \\
Dairy products $\ddagger$ & 3 & 4 \\
Meats, poultry and fish & 3 & 5 \\
Nuts, seeds and legumes & 1 & 2 \\
Fats and oils & 7 & 8 \\
\hline
\end{tabular}

* Data are presented for a energy of $10040 \mathrm{~kJ}(2400 \mathrm{kcal}) / \mathrm{d}$ †At least four servings from whole grains in the DASH diet. ‡Low-fat $(<2 \%)$ in the DASH diet.

\section{Statistical analysis}

To ensure the normal distribution of variables, histogram and Kolmogrov-Smirnov tests were applied. The independent samples Student's $t$ test was used to detect differences in general characteristics and dietary intakes between the two groups. To determine the effect of the DASH diet on metabolic profiles, we applied repeated-measures ANOVA, where treatment $\times$ time interactions were tested by using Pillai's trace. In these analyses, the treatments (DASH and control) were regarded as between-subject factors and time with two time-points (baseline and week 4 of intervention) was considered as a within-subject factor. $P<0.05$ was considered as statistically significant. All statistical analyses were done using the Statistical Package for Social Science version 17 (SPSS, Inc.).

\section{Results}

Mean age of the study participants was not statistically different between the DASH and the control groups. Although pre-pregnancy weight $(75.5(\mathrm{SD} 16 \cdot 3) \quad v .67 \cdot 4(\mathrm{SD} 9 \cdot 3) \mathrm{kg}$, $P=0.08$ ) and BMI (29.6 (SD 5.9) v. 26.7 (SD 3.0$) \mathrm{kg} / \mathrm{m}^{2}$, $P=0.07)$ were higher in controls than that in the DASH diet group, the differences were not statistically significant (Table 2). Baseline weight and BMI, as well as their means after intervention, were not significantly different between individuals who consumed the DASH and control diets.

Based on the $3 \mathrm{~d}$ dietary records that participants provided throughout the study, no statistically significant difference was seen between the two groups in terms of dietary intakes of energy; however, significant differences were found in dietary intakes of SFA, PUFA, cholesterol, dietary fibre, 
Table 2. General characteristics of the study participants

(Mean values and standard deviations)

\begin{tabular}{|c|c|c|c|c|c|}
\hline & \multicolumn{2}{|c|}{$\begin{array}{l}\text { Control diet } \\
\quad(n 17)\end{array}$} & \multicolumn{2}{|c|}{$\begin{array}{l}\text { DASH diet } \\
\quad(n 17)\end{array}$} & \multirow[b]{2}{*}{$P^{*}$} \\
\hline & Mean & SD & Mean & SD & \\
\hline Maternal age (years) & 29.4 & $6 \cdot 2$ & $30 \cdot 7$ & $6 \cdot 7$ & 0.56 \\
\hline Height $(\mathrm{cm})$ & 159.4 & $5 \cdot 3$ & $158 \cdot 7$ & 3.0 & 0.64 \\
\hline Pre-pregnancy weight (kg) & $75 \cdot 5$ & $16 \cdot 3$ & 67.4 & $9 \cdot 3$ & 0.08 \\
\hline Weight at study baseline $(\mathrm{kg})$ & $80 \cdot 0$ & $15 \cdot 8$ & 73.4 & $9 \cdot 3$ & 0.14 \\
\hline Weight at end-of-trial (kg) & $81 \cdot 0$ & $16 \cdot 2$ & $74 \cdot 2$ & $9 \cdot 7$ & 0.14 \\
\hline Pre-pregnancy BMI $\left(\mathrm{kg} / \mathrm{m}^{2}\right)$ & $29 \cdot 6$ & 5.9 & $26 \cdot 7$ & 3.0 & 0.07 \\
\hline BMI at study baseline $\left(\mathrm{kg} / \mathrm{m}^{2}\right)$ & 31.4 & $5 \cdot 7$ & $29 \cdot 0$ & $3 \cdot 2$ & 0.15 \\
\hline $\mathrm{BMI}$ at end-of-trial $\left(\mathrm{kg} / \mathrm{m}^{2}\right)$ & 31.8 & $5 \cdot 9$ & $29 \cdot 4$ & 3.3 & 0.15 \\
\hline
\end{tabular}

DASH, Dietary Approaches to Stop Hypertension.

* Obtained from independent samples Student's $t$ test.

simple sugars, $\mathrm{Na}$ and $\mathrm{K}$ between the two groups $(P<0.05)$ (Table 3).

Adherence to the DASH eating pattern, compared with the control diet, resulted in improved glucose tolerance such that plasma glucose levels reduced at 60 $\left(-1.86 \quad v . \quad-0.45 \mathrm{mmol} / \mathrm{l}, \quad P_{\text {group }}=0.02\right), \quad 120 \quad(-2.3 \quad v$. $\left.0.2 \mathrm{mmol} / \mathrm{l}, P_{\text {group }}=0.001\right)$ and $180 \mathrm{~min}(-1.7 v \cdot 0.22 \mathrm{mmol} / \mathrm{l}$, $\left.P_{\text {group }}=0.002\right)$ after the glucose load (Table 4). Decreased HbA1c levels $\left(-0.2 v .0 .05 \%, P_{\text {group }}=0.001\right)$ were also seen in the DASH group compared with the control group. Mean changes for serum total $(-0.42$ v. $0.31 \mathrm{mmol} / \mathrm{l}$, $\left.P_{\text {group }}=0.01\right)$ and LDL-cholesterol $(-0.47 v .0 .22 \mathrm{mmol} / \mathrm{l}$, $\left.P_{\text {group }}=0.005\right)$, TAG $\left(-0.17 v \cdot 0.34 \mathrm{mmol} / 1, \quad P_{\text {group }}=0.01\right)$ and total:HDL cholesterol ratio ( -0.6 (SD 0.9) v. 0.3 (SD 0.8), $\left.P_{\text {group }}=0.008\right)$ were significantly different between the two diets. Additionally, consumption of the DASH diet favourably influenced systolic blood pressure $(-2.6 v .1 .7 \mathrm{mmHg}$, $\left.P_{\text {group }}=0.001\right)$. Mean changes of fasting plasma glucose $\left(-0.29 v \cdot 0.15 \mathrm{mmol} / 1, P_{\text {group }}=0.09\right)$ were non-significant comparing the DASH diet with the control diet. Although consumption of the DASH diet resulted in increased levels of serum HDL-cholesterol $(0 \cdot 12 \mathrm{mmol} / \mathrm{l})$, we failed to find a significant difference comparing the two diets. The effect of time was significant for plasma glucose levels at 60 $\left(P_{\text {time }}<0.0001\right), 120\left(P_{\text {time }}=0.004\right)$ and $180\left(P_{\text {time }}=0.008\right)$ min after glucose load, as well as for HbA1C $\left(P_{\text {time }}=0.03\right)$ and diastolic blood pressure $\left(P_{\text {time }}=0.009\right)$. No significant time-group interactions were found.

Consumption of the DASH eating pattern, compared with the control diet, resulted in a lower rate of caesarean section (41.2 v. 88.2\%, $P=0 \cdot 001$ ) as well as a decreased need for insulin therapy after delivery $(11.8 v .58 .8 \%, P=0.005)$. Mean birth weight of babies whose mothers received the DASH diet was significantly lower than those whose mothers received the control diet (3083 (SD 402) v. 3641 (SD 579) g, $P=0.006$ ).

\section{Discussion}

We found that consumption of the DASH eating pattern for 4 weeks among pregnant women with GDM had beneficial effects on glucose tolerance, lipid profiles and systolic blood pressure compared with the control diet. However, the effects of the DASH eating pattern on serum HDL-cholesterol and diastolic blood pressure were not significantly different compared with the control diet. To our knowledge, the present study is the first to report the effect of the DASH diet on metabolic profiles of pregnant women with GDM.

Gestational diabetes is associated with several complications in maternal and offspring health, as well as in later stages of their lives ${ }^{(4-6,9)}$. We showed that the DASH eating pattern could significantly improve glucose tolerance and lipid profiles of pregnant women with GDM. Earlier studies have shown the beneficial effects of the DASH eating pattern

Table 3. Dietary intakes of study participants throughout the study

(Mean values and standard deviations)

\begin{tabular}{|c|c|c|c|c|c|}
\hline & \multicolumn{2}{|c|}{$\begin{array}{l}\text { Control diet } \\
\quad(n 17)\end{array}$} & \multicolumn{2}{|c|}{$\begin{array}{l}\text { DASH diet } \\
\quad(n 17)\end{array}$} & \multirow[b]{2}{*}{$P^{*}$} \\
\hline & Mean & SD & Mean & SD & \\
\hline Energy $(\mathrm{kJ} / \mathrm{d})$ & 10016 & 674 & 10050 & 105 & 0.83 \\
\hline Fat $(\mathrm{g} / \mathrm{d})$ & 78 & 15 & 47 & 2.5 & $<0.0001$ \\
\hline Protein $(\mathrm{g} / \mathrm{d})$ & 105 & 17 & 101 & 7 & 0.31 \\
\hline Carbohydrate (g/d) & 323 & 41 & 401 & 13 & $<0.0001$ \\
\hline $\mathrm{SFA}(\mathrm{g} / \mathrm{d})$ & 20.9 & $4 \cdot 1$ & $9 \cdot 2$ & 1.0 & $<0.0001$ \\
\hline PUFA (g/d) & $27 \cdot 2$ & 9.5 & $13 \cdot 7$ & $1 \cdot 1$ & $<0.0001$ \\
\hline Cholesterol $(\mathrm{mg} / \mathrm{d})$ & $178 \cdot 2$ & $45 \cdot 1$ & 94.7 & $27 \cdot 4$ & 0.001 \\
\hline Dietary fibre $(\mathrm{g} / \mathrm{d})$ & $15 \cdot 7$ & $2 \cdot 8$ & $22 \cdot 8$ & 1.6 & $<0.0001$ \\
\hline Insoluble fibre $(\mathrm{g} / \mathrm{d})$ & 3.9 & 1.6 & 5.6 & 0.3 & $<0.0001$ \\
\hline Simple sucrose $(g / d)$ & $19 \cdot 8$ & $1 \cdot 2$ & $9 \cdot 1$ & 0.9 & $<0.0001$ \\
\hline $\mathrm{Na}(\mathrm{mg} / \mathrm{d})$ & $3859 \cdot 0$ & $430 \cdot 4$ & $1378 \cdot 6$ & $192 \cdot 2$ & $<0.0001$ \\
\hline $\mathrm{K}(\mathrm{mg} / \mathrm{d})$ & $2610 \cdot 3$ & $386 \cdot 0$ & $4147 \cdot 3$ & 88.5 & $<0.0001$ \\
\hline $\mathrm{Mg}(\mathrm{mg} / \mathrm{d})$ & $271 \cdot 6$ & $46 \cdot 1$ & 363.9 & $14 \cdot 2$ & $<0.0001$ \\
\hline $\mathrm{Ca}(\mathrm{mg} / \mathrm{d})$ & $1204 \cdot 7$ & $216 \cdot 3$ & $1752 \cdot 0$ & $81 \cdot 0$ & $<0.0001$ \\
\hline Fruit (servings/d) & 3.9 & 0.9 & 5.9 & 0.8 & $<0.0001$ \\
\hline Vegetables (servings/d) & $4 \cdot 0$ & 1.0 & $5 \cdot 8$ & 0.8 & $<0.0001$ \\
\hline Nuts (servings/d) & 0.4 & 0.1 & 1.9 & 0.2 & $<0.0001$ \\
\hline Fats and oils (servings/d) & 4.0 & 0.5 & $2 \cdot 0$ & 0.5 & $<0.0001$ \\
\hline
\end{tabular}

* Obtained from independent samples Student's $t$ test. 


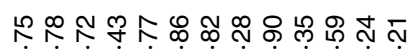

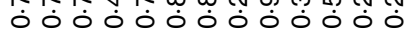

* $\underline{\xi}$

옹

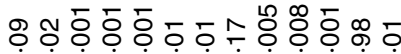

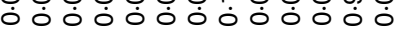

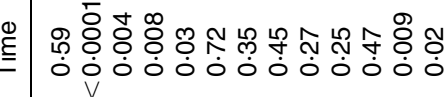

ஓ ம

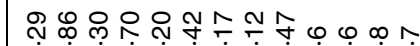

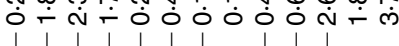

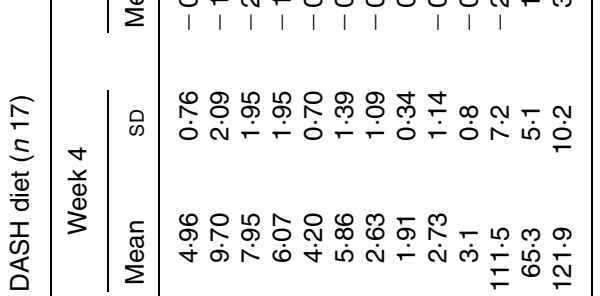
i

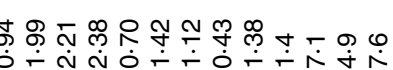

$\stackrel{2}{\stackrel{2}{\Phi}}$

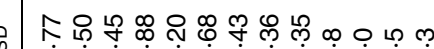

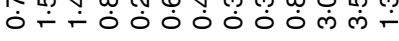

on fasting plasma glucose and lipid profiles of patients with hypertension, CVD, the metabolic syndrome and T2 $\mathrm{D}^{(15,16,19,20)}$. In a study by Azadbakht et al. ${ }^{(15)}$, a significant reduction in body weight, waist circumference, fasting blood glucose levels, \%HbA1 $1_{\mathrm{C}}$, serum LDL-cholesterol levels, and in systolic and diastolic blood pressure was found with the DASH diet that was consumed for 8 weeks among type 2 diabetic patients. Adherence to the DASH diet for 8 weeks among men and women with the metabolic syndrome resulted in similar findings ${ }^{(16)}$. Harsha et al. ${ }^{(21)}$ found favourable effects of the DASH diet, compared with the control diet, on serum total and LDL-cholesterol levels in subjects with elevated blood pressure. They concluded that the beneficial effects of the DASH eating pattern on serum lipid profiles is independent of the dietary $\mathrm{Na}$ levels of this diet. The same findings on serum lipid profiles have also been reported among hypercholesterolaemic patients ${ }^{(22)}$. The present findings are in line with previously reported ones on the beneficial effects of the DASH diet on metabolic profiles. It must be kept in mind that the control diet in the present study resulted in increased serum TAG and LDL-cholesterol levels, as well as systolic and diastolic blood pressure from baseline. One might assume that this was due to the design of the control diet or to some other reasons, including bias in advice. We think that the increments seen in serum TAG and LDLcholesterol levels, as well as in SBP and DBP, are due to the design of the control diet which contains lower amounts of whole grains, vegetables and higher amounts of simple sugars compared with the DASH diet. However, it is very difficult to understand the exact reason for this finding. Although the control diet has been planned based on 'usual advice' given to GDM patients in clinical situations, the diet was delivered by the one who was responsible for delivering the DASH diet as well. The use of only one dietitian to deliver the control and intervention diets might be a source of potential bias, because that person can load the dice in favour of the intervention diet. Definitely, this clinical trial would have been better if the 'usual advice' given to patients with GDM in the control group had been delivered by a separate person. However, the components of the diets were different. Moreover, the dietitian was responsible for delivering the menus, and the $7 \mathrm{~d}$ menu cycle was planned by the study dietitian and re-checked by the study senior investigator. However, the importance of metabolic control in GDM patients is of great importance due to the unfavourable effects of GDM on pregnancy outcome.

Several mechanisms can explain the health benefits of the DASH eating pattern. High content of dietary fibre and phyto-oestrogens due to higher amounts of fruit and vegetables in the DASH diet might contribute to its beneficial effects on serum TAG, total and LDL-cholesterol levels ${ }^{(16,22,23)}$. Furthermore, the simple sugar content of the DASH diet was almost half that of the control diet. Earlier studies have reported that a high-sucrose diet increases serum glucose and lipid profiles ${ }^{(24-26)}$. Consumption of high-GI carbohydrates in the control diet pattern could result in increased levels of plasma glucose concentrations, whereas a low-GI DASH pattern would result in better metabolic control. Earlier studies have shown that consuming 
a high-carbohydrate diet that is mainly composed of low-GI varieties can maintain blood glucose concentrations within normal ranges ${ }^{(12,27,28)}$.

Increasing dietary intakes of soluble fibre has also been shown to reduce serum total cholesterol concentrations, independent of dietary fat intake ${ }^{(2930)}$. High intake of legumes in the DASH diet, compared with the control diet, might also be responsible for its beneficial effects on glucose tolerance and lipid profiles ${ }^{(31)}$. In a study by Azadbakht et al. ${ }^{(32)}$, soya consumption in patients with T2D with nephropathy reduced total cholesterol, TAG and LDL-cholesterol levels. Such findings have also been reported in hypercholesterolaemic patients ${ }^{(33)}$. Consuming higher amounts of non-hydrogenated vegetable oils in the DASH diet, compared with the control diet, might also contribute to favourable effects on glucose tolerance and improved lipid profiles. Several studies have reached modulation of blood pressure and serum lipid profiles with consumption of edible vegetable oils ${ }^{(34,35)}$. In the present study, $\mathrm{Na}$ intake in the control diet was 2.8 times greater than that in the DASH diet. Several studies reported that high $\mathrm{Na}$ intake was associated with abnormal metabolic profiles and cardiovascular risks ${ }^{(36,37)}$. High $\mathrm{Na}$ intake could result in abnormal metabolic profiles through activation of the renin-angiotensin-aldosterone system $^{(38)}$, G972R polymorphism of the IRS-1 gene related to insulin resistance ${ }^{(39)}$, increased signalling through the mineralocorticoid receptor and increased production of reactive oxygen species and oxidative stress, which in turn contribute to insulin resistance ${ }^{(40)}$. Further beneficial effects of the DASH diet, compared with the control diet, could be explained by higher $\mathrm{K}$ intake in the DASH diet. $\mathrm{K}$ intake in the DASH group was almost 1.5 times greater than that in the control group. Recent studies have indicated that high $\mathrm{K}$ intake can result in improved insulin resistance and lipid profiles ${ }^{(41,42)}$. Although the precise mechanisms of beneficial effects of dietary $\mathrm{K}$ on metabolic profiles have not been fully elucidated, it could exert its effects through sympathetic nerve inhibition, suppressed salt-induced insulin resistance and decreased production of reactive oxygen species $^{(43)}$. High intake of $\mathrm{Mg}$ and $\mathrm{Ca}$ in the DASH diet, compared with the control diet, might also be responsible for improving glucose tolerance and decreased lipid profiles. Earlier studies have reported that increased dietary $\mathrm{Mg}$ intake ameliorates insulin resistance, serum lipid profiles and lowers inflammation, endothelial dysfunction, oxidative stress and platelet aggregability ${ }^{(19,44)}$. Such findings have also been reached with high $\mathrm{Ca}$ intake. Higher $\mathrm{Ca}$ intake would lead to decreased fatty acid absorption and increased faecal fatty acid content ${ }^{(45)}$. This reduced absorption of fatty acids could in turn result in decreased levels of metabolic profiles ${ }^{(46)}$. Furthermore, increasing dietary Ca intake can result in an elevation of liver intracellular $\mathrm{Ca}$, which in turn would stimulate microsomal TAG transfer $\operatorname{protein}^{(47)}$. Probably, microsomal TAG transfer protein can lower the formation and secretion of lipid profiles.

One of the limitations of the present study is the duration of this trial. We were unable to administer the diets for more than 4 weeks due to the particular condition of the pregnant women.
In conclusion, consumption of the DASH eating pattern for 4 weeks among pregnant women with GDM results in beneficial effects on glucose tolerance and lipid profiles compared with a control diet.

\section{Acknowledgements}

The present study was supported by a grant (no. 9013) from the Vice-chancellor for Research, KUMS, Kashan, Iran. The authors would like to thank the staff of Naghavi and Shaheed Beheshti Clinics (Kashan, Iran) for their assistance in the present project. Z. A. conducted the study, carried out the statistical analyses, wrote the manuscript and contributed in the interpretation of the findings. Z. T., M. S. and T. F. contributed in data collection and assisted in writing the manuscript. A. E. contributed in conception and design and also advised on statistical analyses, contributed in drafting the manuscript and assisted in interpretation of the findings. All authors approved the final version of the manuscript. The authors declare no conflict of interest.

\section{References}

1. Wang Y, Nie M, Li W, et al. (2011) Association of six single nucleotide polymorphisms with gestational diabetes mellitus in a Chinese population. PLoS One 6, e26953.

2. Shaat N \& Groop L (2007) Genetics of gestational diabetes mellitus. Curr Med Chem 14, 569-583.

3. Metzger BE, Buchanan TA, Coustan DR, et al. (2007) Summary and recommendations of the Fifth International Workshop-Conference on Gestational Diabetes Mellitus. Diabetes Care 30, Suppl. 2, S251-S260.

4. Bener A, Saleh NM \& Al-Hamaq A (2011) Prevalence of gestational diabetes and associated maternal and neonatal complications in a fast-developing community: global comparisons. Int J Womens Health 3, 367-373.

5. Gabbe SG \& Graves CR (2003) Management of diabetes mellitus complicating pregnancy. Obstet Gynecol 102, 857-868.

6. Jelsema RD (2004) Management of diabetes mellitus complicating pregnancy. Obstet Gynecol 103, 586 (author reply 586-587).

7. Yessoufou A \& Moutairou K (2011) Maternal diabetes in pregnancy: early and long-term outcomes on the offspring and the concept of "metabolic memory". Exp Diabetes Res 2011, 218598.

8. Cox NJ (1994) Maternal component in NIDDM transmission. How large an effect? Diabetes 43, 166-168.

9. Linne Y, Barkeling B \& Rossner S (2002) Natural course of gestational diabetes mellitus: long term follow up of women in the SPAWN study. BJOG 109, 1227-1231.

10. Acheson KJ (2010) Carbohydrate for weight and metabolic control: where do we stand? Nutrition 26, 141-145.

11. Lim SS, Noakes M \& Norman RJ (2007) Dietary effects on fertility treatment and pregnancy outcomes. Curr Opin Endocrinol Diabetes Obes 14, 465-469.

12. McGowan CA \& McAuliffe FM (2010) The influence of maternal glycaemia and dietary glycaemic index on pregnancy outcome in healthy mothers. Br J Nutr 104, 153-159.

13. Moses RG, Barker M, Winter M, et al. (2009) Can a low-glycemic index diet reduce the need for insulin in gestational diabetes mellitus? A randomized trial. Diabetes Care 32 , 996-1000. 
14. Vollmer WM, Sacks FM, Ard J, et al. (2001) Effects of diet and sodium intake on blood pressure: subgroup analysis of the DASH-sodium trial. Ann Intern Med 135, 1019-1028.

15. Azadbakht L, Fard NR, Karimi M, et al. (2011) Effects of the Dietary Approaches to Stop Hypertension (DASH) eating plan on cardiovascular risks among type 2 diabetic patients: a randomized crossover clinical trial. Diabetes Care $\mathbf{3 4}$, 55-57.

16. Azadbakht L, Mirmiran P, Esmaillzadeh A, et al. (2005) Beneficial effects of a Dietary Approaches to Stop Hypertension eating plan on features of the metabolic syndrome. Diabetes Care 28, 2823-2831.

17. Jehan I, Zaidi S, Rizvi S, et al. (2010) Dating gestational age by last menstrual period, symphysis-fundal height, and ultrasound in urban Pakistan. Int $J$ Gynaecol Obstet 110, $231-234$

18. Rossi G (2010) Diagnosis and classification of diabetes mellitus. Recenti Prog Med 101, 274-276.

19. Champagne CM (2008) Magnesium in hypertension, cardiovascular disease, metabolic syndrome, and other conditions: a review. Nutr Clin Pract 23, 142-151.

20. Doyle L \& Cashman KD (2003) The effect of nutrient profiles of the Dietary Approaches to Stop Hypertension (DASH) diets on blood pressure and bone metabolism and composition in normotensive and hypertensive rats. Br J Nutr $\mathbf{8 9}$, 713-724.

21. Harsha DW, Sacks FM, Obarzanek E, et al. (2004) Effect of dietary sodium intake on blood lipids: results from the DASH-sodium trial. Hypertension 43, 393-398.

22. Obarzanek E, Sacks FM, Vollmer WM, et al. (2001) Effects on blood lipids of a blood pressure-lowering diet: the Dietary Approaches to Stop Hypertension (DASH) Trial. Am J Clin Nutr 74, 80-89.

23. Sacks FM \& Katan M (2002) Randomized clinical trials on the effects of dietary fat and carbohydrate on plasma lipoproteins and cardiovascular disease. Am J Med 113, Suppl. 9B, 13S-24S.

24. Macan M, Vrkic N, Vrdoljak AL, et al. (2010) Effects of high sucrose diet, gemfibrozil, and their combination on plasma paraoxonase 1 activity and lipid levels in rats. Acta Biochim Pol 57, 321-326.

25. Shikany JM, Phadke RP, Redden DT, et al. (2009) Effects of low- and high-glycemic index/glycemic load diets on coronary heart disease risk factors in overweight/obese men. Metabolism 58, 1793-1801.

26. Man Q \& He L (2009) Effects of high-sugar and high-fat diet on growth and carbohydrate, lipid metabolism in Wistar rats. Wei Sheng Yan Jiu 38, 722-724.

27. Scholl TO, Chen X, Khoo CS, et al. (2004) The dietary glycemic index during pregnancy: influence on infant birth weight, fetal growth, and biomarkers of carbohydrate metabolism. Am J Epidemiol 159, 467-474.

28. Romon M, Nuttens MC, Vambergue A, et al. (2001) Higher carbohydrate intake is associated with decreased incidence of newborn macrosomia in women with gestational diabetes. J Am Diet Assoc 101, 897-902.

29. Van Horn L (1997) Fiber, lipids, and coronary heart disease. A statement for healthcare professionals from the Nutrition Committee, American Heart Association. Circulation 95, 2701-2704.

30. Brown L, Rosner B, Willett WW, et al. (1999) Cholesterollowering effects of dietary fiber: a meta-analysis. Am J Clin Nutr 69, 30-42.
31. Esmaillzadeh A \& Azadbakht L (2012) Legume consumption is inversely associated with serum concentrations of adhesion molecules and inflammatory biomarkers among Iranian women. J Nutr 142, 334-339.

32. Azadbakht L, Shakerhosseini R, Atabak S, et al. (2003) Beneficiary effect of dietary soy protein on lowering plasma levels of lipid and improving kidney function in type II diabetes with nephropathy. Eur J Clin Nutr 57, 1292-1294.

33. Hermansen K, Hansen B, Jacobsen R, et al. (2005) Effects of soy supplementation on blood lipids and arterial function in hypercholesterolaemic subjects. Eur J Clin Nutr 59, $843-850$.

34. Sudhakar B, Kalaiarasi P, Al-Numair KS, et al. (2011) Effect of combination of edible oils on blood pressure, lipid profile, lipid peroxidative markers, antioxidant status, and electrolytes in patients with hypertension on nifedipine treatment. Saudi Med J 32, 379-385.

35. Esmaillzadeh A \& Azadbakht L (2011) Different kinds of vegetable oils in relation to individual cardiovascular risk factors among Iranian women. BrJ Nutr 105, 919-927.

36. Alderman MH \& Cohen HW (2012) Dietary sodium intake and cardiovascular mortality: controversy resolved? Curr Hypertens Rep 14, 193-201.

37. Gonzalez SA, Forcada P, de Cavanagh EM, et al. (2012) Sodium intake is associated with parasympathetic tone and metabolic parameters in mild hypertension. Am J Hypertens 25, 620-624.

38. Huan Y, Deloach S, Keith SW, et al. (2012) Aldosterone and aldosterone: renin ratio associations with insulin resistance and blood pressure in African Americans. J Am Soc Hypertens 6, 56-65.

39. Dziwura J, Binczak-Kuleta A, Miazgowski T, et al. (2011) The associations between G972R polymorphism of the IRS-1 gene, insulin resistance, salt sensitivity and non-dipper hypertension. Hypertens Res 34, 1082-1086.

40. Lastra G, Dhuper S, Johnson MS, et al. (2010) Salt, aldosterone, and insulin resistance: impact on the cardiovascular system. Nat Rev Cardiol 7, 577-584.

41. Ishikawa M, Arai S, Takano M, et al. (2010) Taurine's health influence on Japanese high school girls. J Biomed Sci 17, Suppl. 1, S47.

42. Reungjui S, Pratipanawatr T, Johnson RJ, et al. (2008) Do thiazides worsen metabolic syndrome and renal disease? The pivotal roles for hyperuricemia and hypokalemia. Curr Opin Nephrol Hypertens 17, 470-476.

43. Ando K, Matsui H, Fujita M, et al. (2010) Protective effect of dietary potassium against cardiovascular damage in salt-sensitive hypertension: possible role of its antioxidant action. Curr Vasc Pharmacol 8, 59-63.

44. Bo S \& Pisu E (2008) Role of dietary magnesium in cardiovascular disease prevention, insulin sensitivity and diabetes. Curr Opin Lipidol 19, 50-56.

45. Reid IR (2004) Effects of calcium supplementation on circulating lipids: potential pharmacoeconomic implications. Drugs Aging 21, 7-17.

46. Vaskonen T (2003) Dietary minerals and modification of cardiovascular risk factors. J Nutr Biochem 14, 492-506.

47. Cho HJ, Kang HC, Choi SA, et al. (2005) The possible role of $\mathrm{Ca}^{2+}$, on the activation of microsomal triglyceride transfer protein in rat hepatocytes. Biol Pharm Bull 28, 1418-1423. 